\title{
FIBRE: a broadband submillimeter spectrometer using superconducting bolometer arrays
}

Johannes G. Staguhn, Dominic J. Benford, Francois Pajot, Troy Ames, Christine A. Allen, et al.

Johannes G. Staguhn, Dominic J. Benford, Francois Pajot, Troy Ames, Christine A. Allen, James A. Chervenak, Sebastien Lefranc, Steven Maher, S. Harvey Moseley, Thomas Phillips, Cyrille G. Rioux, Richard A. Shafer, George M. Voellmer, "FIBRE: a broadband submillimeter spectrometer using superconducting bolometer arrays," Proc. SPIE 5498, Millimeter and Submillimeter Detectors for Astronomy II, (8 October 2004); doi: $10.1117 / 12.552128$

Event: SPIE Astronomical Telescopes + Instrumentation, 2004, Glasgow, United Kingdom 


\title{
FIBRE: A broadband submillimeter spectrometer using superconducting bolometer arrays
}

\author{
Johannes Staguhn ${ }^{a, b}$, Dominic Benford ${ }^{a}$, François Pajot ${ }^{c}$, Troy Ames ${ }^{a}$, Christine Allen ${ }^{a}$, \\ Jay Chervenak $^{a}$, Sebastien Lefranc ${ }^{c}$, Steven Maher ${ }^{a, b}$, Harvey Moseley ${ }^{a}$, Thomas Phillips ${ }^{d}$, Rioux, \\ C., Rick Shafer ${ }^{a}$, George Voellmer ${ }^{a}$ \\ ${ }^{a}$ NASA/Goddard Space Flight Center \\ ${ }^{b}$ SSAI, Lanham, MD \\ ${ }^{c}$ Institut d'Astrophysique Spatiale \\ ${ }^{d}$ Caltech
}

\begin{abstract}
The Fabry-Perot Interferometer Bolometer Research Experiment FIBRE, a protoype submillimeter spectrometer for astronomical observations, is based on a helium-cooled scanning Fabry-Perot and superconducting transition edge sensor bolometers (TES). The TES design takes advantage of a recently discovered method of excess noise reduction by depositing lateral normal metal bars on these devices. A SQUID multiplexer is used to read out the individual detector pixels. The spectral resolving power of the instrument is provided by a Fabry-Perot spectrometer. The outgoing light from the Fabry-Perot passes onto a low resolution grating for order sorting. A linear bolometer array consisting of 16 elements detects this dispersed light, capturing 5 orders simultaneously from one position on the sky. With tuning of the Fabry-Perot over one free spectral range, a spectrum covering $\Delta \lambda / \lambda=1 / 7$ at a resolution of $\sim 1 / 1200$ can be achieved. This spectral resolution is sufficient to resolve doppler broadened line emission from external galaxies. FIBRE operates in the $350 \mu \mathrm{m}$ and $450 \mu \mathrm{m}$ bands. These bands cover line emission from the important PDR tracers neutral carbon [CI] and carbon monoxide CO. The spectrometer is used at the Caltech Submillimeter Observatory for astronomical observations.
\end{abstract}

Keywords: detectors, bolometers, multiplexers, sub-mm instruments, spectrometers

\section{INTRODUCTION}

Sensitive broadband submillimeter and far infrared (FIR) spectral line observations allow insight into the physical nature of star forming regions in external galaxies. The required large bandwidth combined with the low photon energies requires low noise detector technology. There are two fundamentally different technologies available for observations at these frequencies: Heterodyne instruments (with mixers usually consisting of SIS Josephson Junctions at frequencies below $1 \mathrm{THz}$ and hot electron bolometers (HEB) at higher frequencies) provide excellent spectroscopic capabilities, but they suffer from bandwidth limitations and they have a limited noise performance at higher frequencies. The fundamental quantum noise of heterodyne instruments in units of Kelvin is $h v / k$ and therefore increases linearly with frequency. The quantum limit of heterodyne instruments, combined with the limited bandwidth of existing backends imposes strong constraints of these devices on the sensitivity near and above $\mathrm{THz}$ frequencies. There is no such fundamental quantum limit for inncoherent bolometer detectors, which are the second class of detectors working at these frequencies. These wavelength insensitive devices are limited by thermal noise, which can be controlled by the detector design and temperature of operation, allowing detector designs with significantly lower noise than heterodyne instruments. Bolometers are therefore the best choice for sub-mm and FIR continuum observations. For spectral line observations however, efficiency consideration often still favor heterodyne instruments for high spectral resolution applications. Consequently most of the first generation of bolometer instruments for the (sub-) $\mathrm{mm}$ regime, (e.g. SCUBA [1], SHARC [2], both operating at sub-mm wavelengths, and MAMBO [3] operating at mm wavelengths) are continuum devices.

The Fabry-Perot Bolometer Interferometer Experiment, FIBRE, is an instrument designed to demonstrate a suite of advanced technologies, suitable for sensitive spectroscopic detection of far-infrared light. This includes superconducting transition edge sensor (TES) bolometers [4], multiplexed SQUID amplifiers [5], and a cryogenic Fabry-Perot 
interferometer for moderate resolution spectroscopy. These components are being developed for the SOFIA imaging Fabry-Perot spectrometer SAFIRE [6] and for a complement of ground-based instruments. FIBRE allows broad band $\Delta \lambda / \lambda=1 / 7(\sim 60 \mathrm{GHz})$ low resolution $\Delta \lambda / \lambda=1 / 1200(\sim 650 \mathrm{MHz})$ observations covering the $450 \mu \mathrm{m}$ and $350 \mu \mathrm{m}$ atmospherical windows. Fine structure lines of neutral carbon $[\mathrm{CI}]$ and rotational lines of $\mathrm{CO}$, all of which are important tracers of the interstellar medium and of the physical conditions in photo dissociation regions (PDR) are found in these windows. FIBRE's spectral resolution corresponds to a velocity resolution of about $250 \mathrm{~km} / \mathrm{s}$, which is well suited to resolve molecular line emission from external galaxies. The spectral resolving power was chosen, because the main scientific goals are observations of emission lines from a variety of extraglalctic sources. The large bandwidth combined with the sensitivity furthermore allows measurements of the underlying continuum emission. Figure 1 shows a synthesized spectrum of M82 with FIBRE's spectral resolution. Both, the $\mathrm{CO}(\mathrm{J}=7-6)$ and $[\mathrm{CI}]\left({ }^{3} \mathrm{P}_{2}{ }^{3} \mathrm{P}_{1}\right)$ spectral lines are within this band. Note the offset and the slope in the underlying continuum emission.

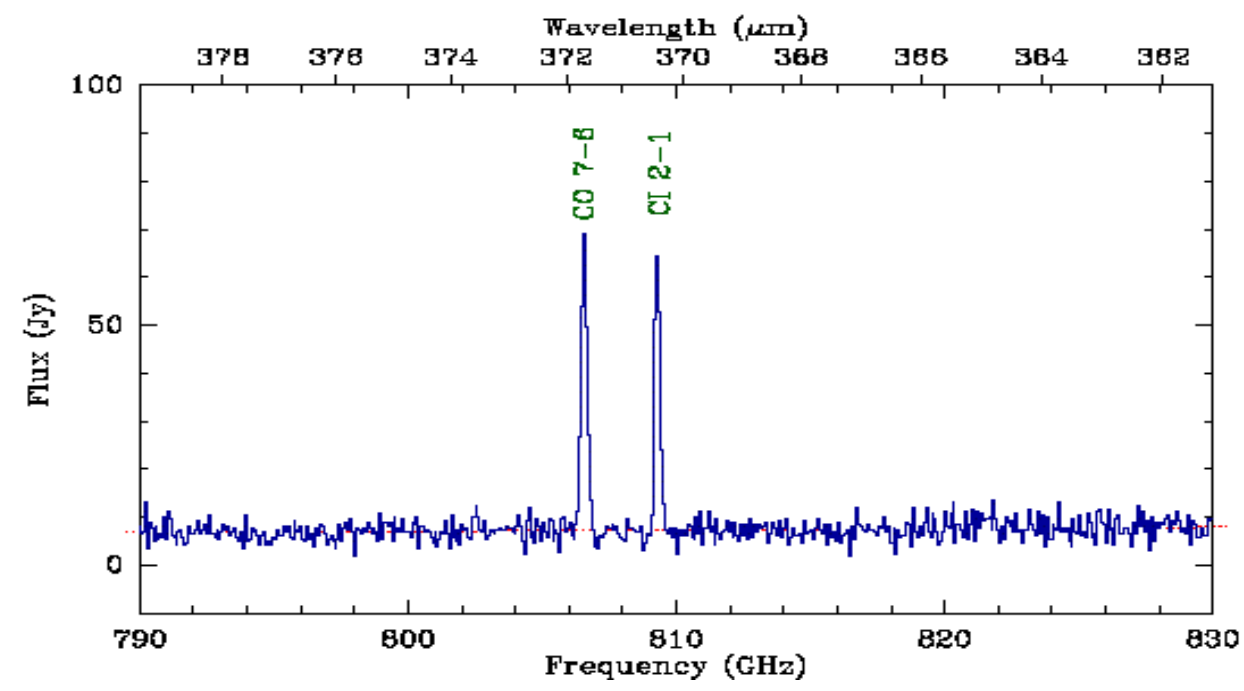

Figure 1. Simulated spectrum of the starforming galaxy M82 with FIBRE's spectral resolution. The CO(J=7-6) and [CI] $\left({ }^{3} \mathrm{P}_{2}-{ }^{3} \mathrm{P}_{1}\right)$ spectral lines as well as the level and slope of the dust continuum emission can be observed.

\section{THE FIBRE INSTRUMENT}

FIBRE uses superconducting Transition Edge Sensor bolometers, which were developed for observations ranging from $\mathrm{X}$-ray to $\mathrm{mm}$ wavelength. TES have a number of advantages over semiconductor bolometers. Voltage biased TES are kept on the transition by the "electrothermal feedback". This self regulating effect which leads to the result that the power dissipation in the TES due to electrical power plus the optical power from the incident photons is constant over a wide range of photon loads and increases the intrinsic speed of the bolometer [7]. The range of optical power over which a TES can be biased is determined by the difference between the transition temperature and the cold head temperature and the thermal link of the TES to it's heat sink. A multiplexed readout of I-V curves from our current FIBRE detectors [8] (including one reference open "dark" reference channel) is shown in Fig. 2 (left). Figure 2 (right) shows the response of these detectors to an external chopped hot-cold load signal.

The TES in FIBRE have a transition temperature of $440 \mathrm{mK}$. The detector package is cooled to $300 \mathrm{mK}$. SQUID amplifiers provide a large noise margin and thus allow multiplexed readout. Staguhn et al. [9] show that the NIST designed SQUID multiplexers allow detector noise limited readout for our TES arrays. FIBRE features two 1 by 8 monolithic bolometer arrays consisting of $1 \mathrm{~mm}$ by $1 \mathrm{~mm}$ absorbers with a $200 \mu \mathrm{m}$ by $50 \mu \mathrm{m} \mathrm{Mo/Au} \mathrm{bilayer} \mathrm{TES.} \mathrm{Fig.} 3$ shows a photograph of a 1x8 planar array. The TES bolometers in our new $1 \times 8$ arrays have transversal metal bar deposition onto the $\mathrm{Mo} / \mathrm{Au}$ bilayer. This design provides reduced out of band excess noise [8]. Figure 4 shows an 8 channel NIST designed SQUID multiplexer with integrated "series array" pre-amplifier, consisting of 100 dc SQUID in series. 
The optical design uses a single Fabry-Perot etalon followed by an order sorting grating. The grating is blazed to operate in one grating order, where up to 6 of the Fabry-Perot orders are transmitted to the detector arrays with a dispersion of approximately $5 \mu \mathrm{m} / \mathrm{mm}$ (Figs. $5 \& 6$ ). In this manner, a spectrum consisting of several orders of the Fabry-Perot is collected instantaneously. The illumination for a closed packed array of 24 detector elements in FIBRE is shown in Fig. 2. In our observing run, the pixels in Fig. 2, labeld 1-2, 11-14, 23-24 were void fields. By stepping the Fabry-Perot over one free spectral range, a complete spectrum with a resolution of 1200 is accumulated over a bandwidth of about $60 \mathrm{GHz}$. Figure 4 shows FIBRE's optical setup.
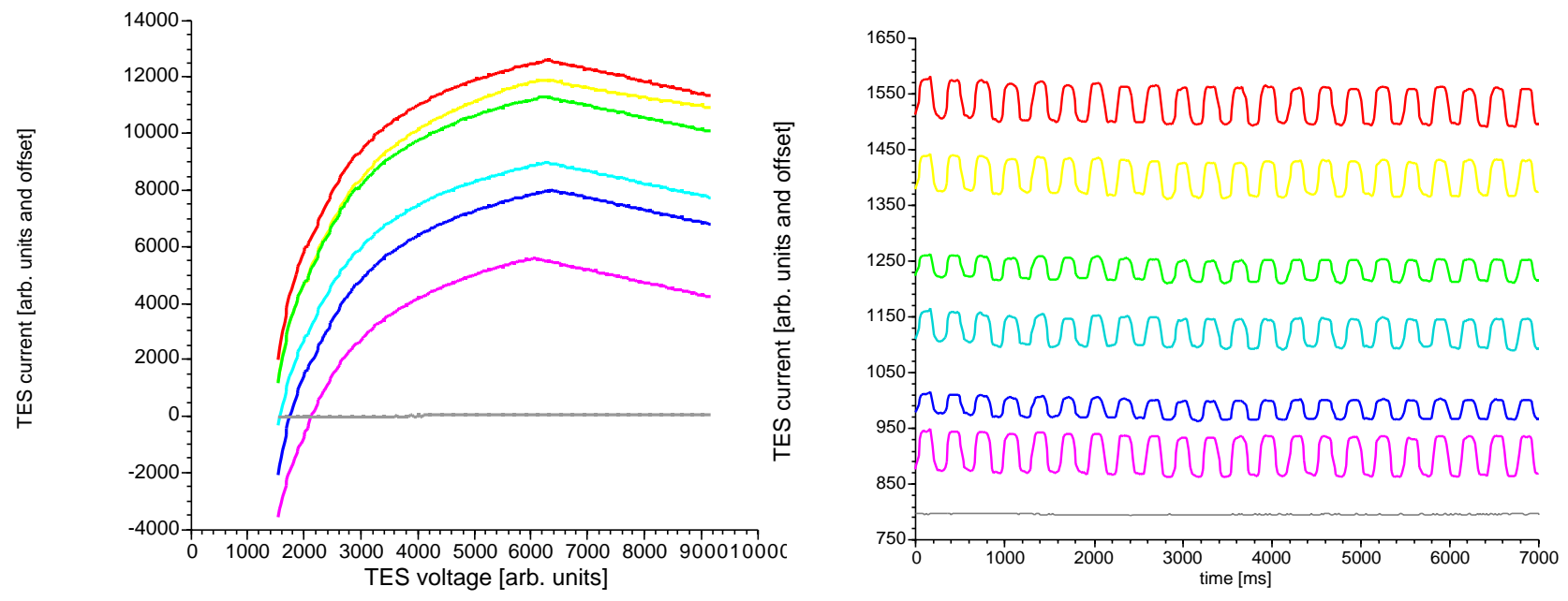

Figure 2. left: I-V curves of new FIBRE TES detectors, including one reference "dark" pixel. The falling part of the curve is the range on the transition where the dissipated electrical power is constant, i.e we have the hyperbolic dependency of I over $\mathrm{U}$ with $\mathrm{I}=$ const/U. The detector goes normal for the highest values of bias voltages. right:

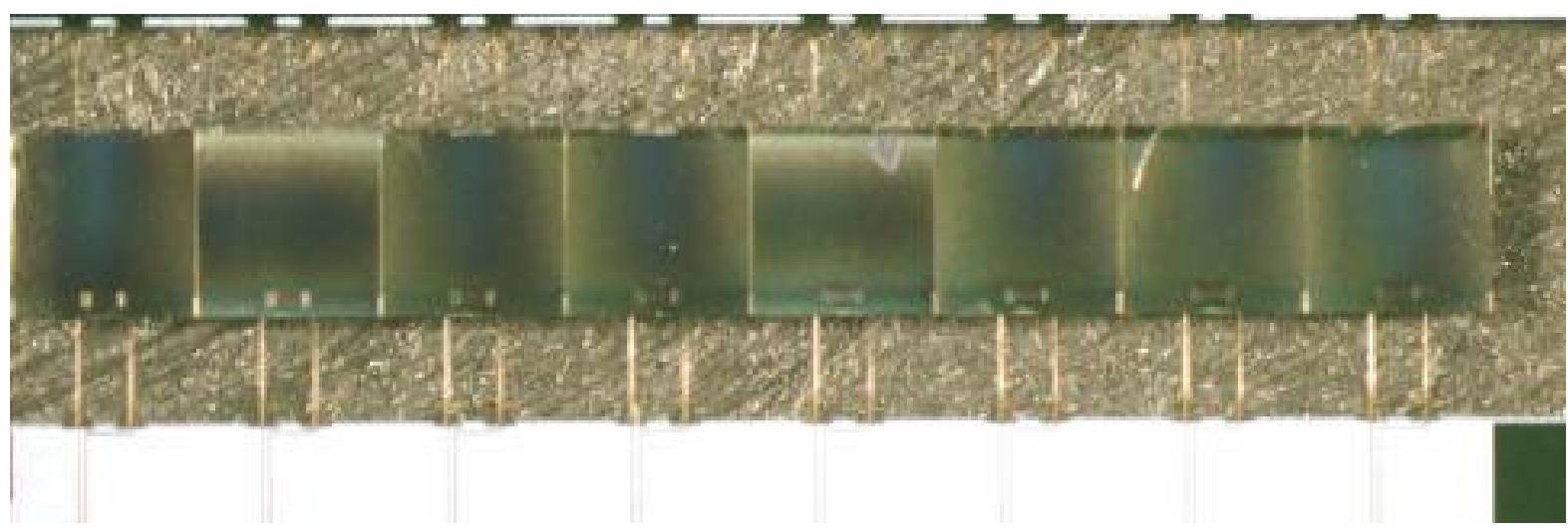

FIGURE 3. Photograph of a single 1 by 8 monolithic bolometer array. The $1 \mathrm{~mm}$ by $1 \mathrm{~mm}$ pixels are $1 \mu \mathrm{m}$ thick silicon membranes supported by 4 legs approximately $5 \mu \mathrm{m}$ wide. The TES is the small pale rectangle at the bottom center of each detector. 


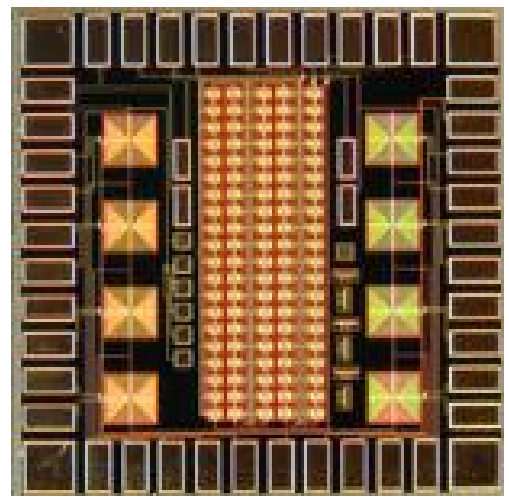

FIGURE 4. Photograph of the NIST designed 1 by 8 SQUID multiplexer used in FIBRE for the TES readout. The size of the chip is $\sim 6 \mathrm{~mm}$ by $6 \mathrm{~mm}$. The 8 square shaped devices are the input and the feedback coils for the 8 first stage SQUIDS. The 100 squares in the center the $2^{\text {nd }}$ stage "series array" consisting of 100 series SQUIDS which act as preamplifiers.

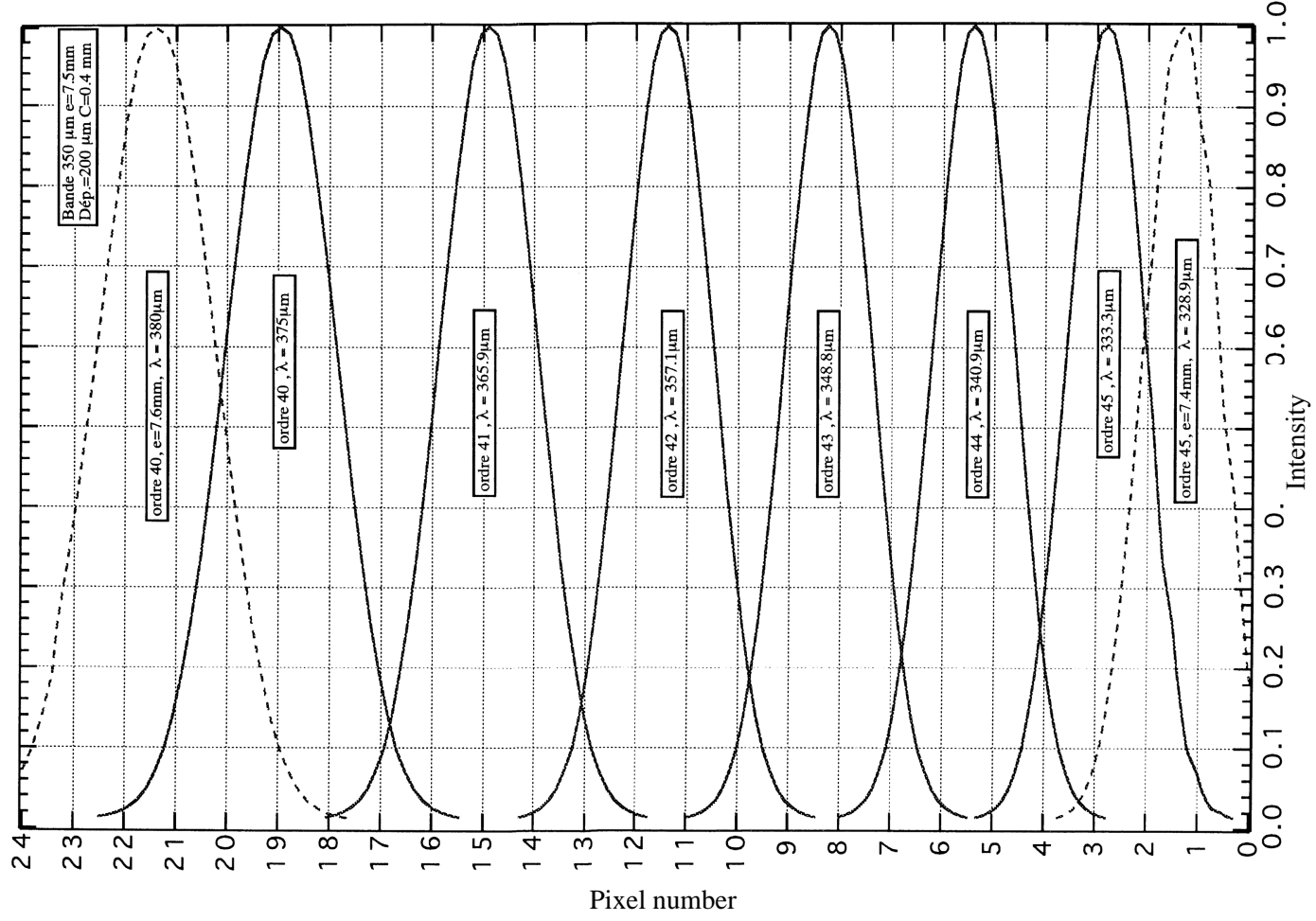

Figure 5. Illumination pattern of the detector array in FIBRE, here for a closed packed detector array with 24 elements. The actual 16 pixel coverage in the current FIBRE setup ranges from pixel number 2 through 9 and 14 through 21 on the figure shown here. 


\section{COMISSIONING RUN AT THE CALTECH SUBMILLIMETER OBSERVATORY}

FIBRE was commissioned at the Caltech Submillimeter Observatore (CSO) on Mauna Kea in Hawaii during an observing run in May/June 2001 (Fig. 7). The instrument was set up to observe in the atmospheric $350 \mu \mathrm{m}$ window. Initial test measurements with the CSO local oscillator verified the optical performance in multiplexed readout mode (Fig. 8). Load measurements were used to derive the detector- as well as the optical- performance of the instrument on the telescope. The detector dark NEP was $\sim 8 \times 10^{-17} \mathrm{~W} / \mathrm{Hz}^{1 / 2}$. This corresponds to an rms noise of $160 \mathrm{mK} \mathrm{s} \mathrm{s}^{-1 / 2}$ at the entrance of the FIBRE dewar. The system temperature (outside the dewar) of FIBRE during the run corresponds to a radiometric noise temperature of $800 \mathrm{~K}$. The main cause for this relatively high value is the low optical efficiency of $4 \%$, combined with the field stop to detector couplig factor, which is $19 \%$.

The 6 nights of observing time suffered from poor weather conditions. The best $350 \mu \mathrm{m}$ opacity we observed was a $\tau$ of 3 , typically the opacity was at $\tau \sim 4$. Figure 9 (left) shows a skydip observed with FIBRE. The FIBRE skydips are well in agreement with the values derived from the observatory's $\tau$-meter. Despite the poor weather conditions, we managed to observe the limb of the moon. A scan over the edge of the moon is displayed in Fig. 9 (right). Each of the data points shown in the figure corresponds to an on source integration of 3 seconds. These observations represent the first astronomical observations with TES bolometers in the sub-mm regime and the first astronomical observations ever with multiplexed readout of TES detectors.
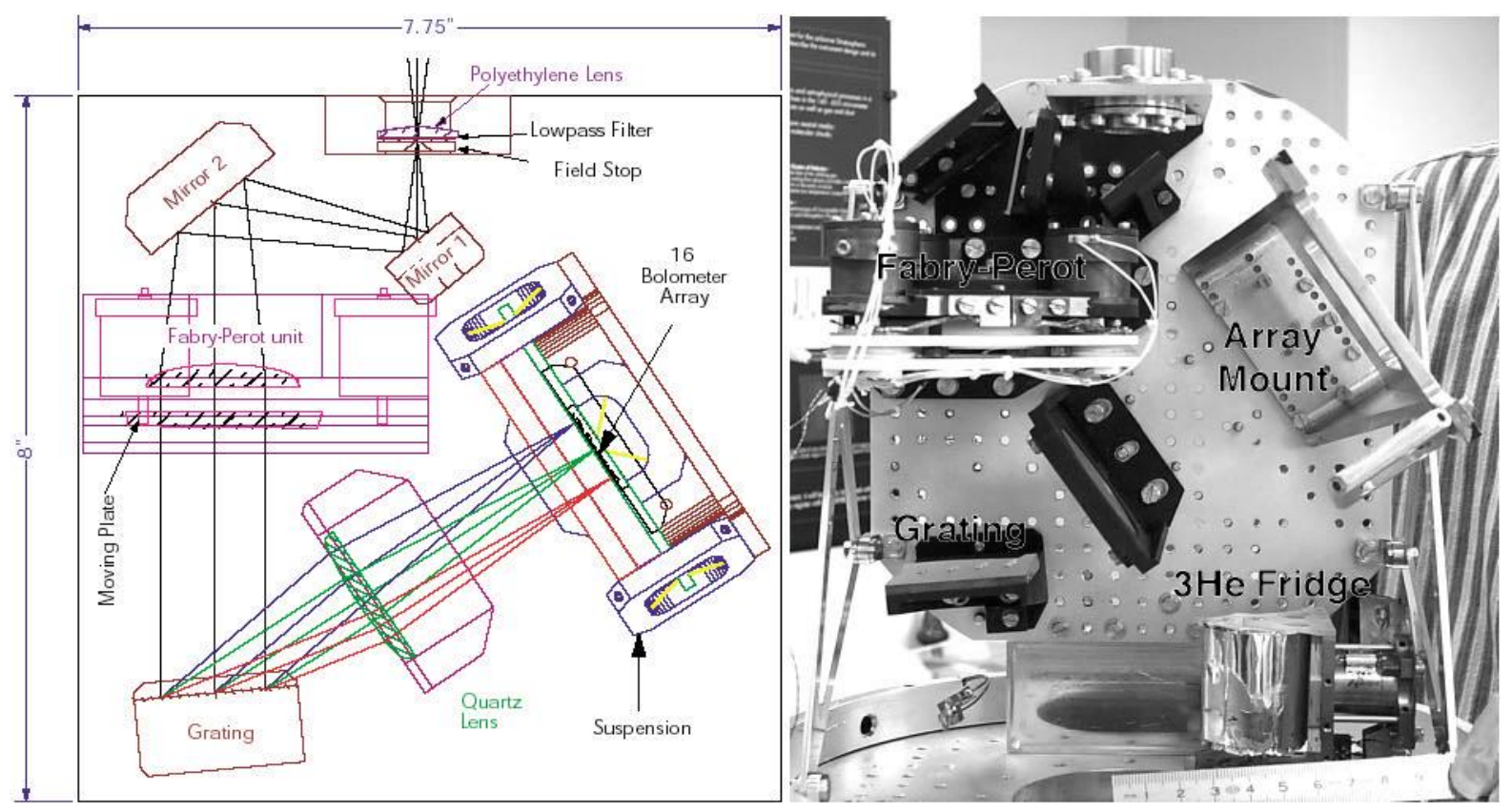

Figure 6. (Left) Diagram of spectrometer optics,showing three orders being dispersed onto the bolometer array.(Right)Optics being assembled,with the detector array and baffles yet to be added. 


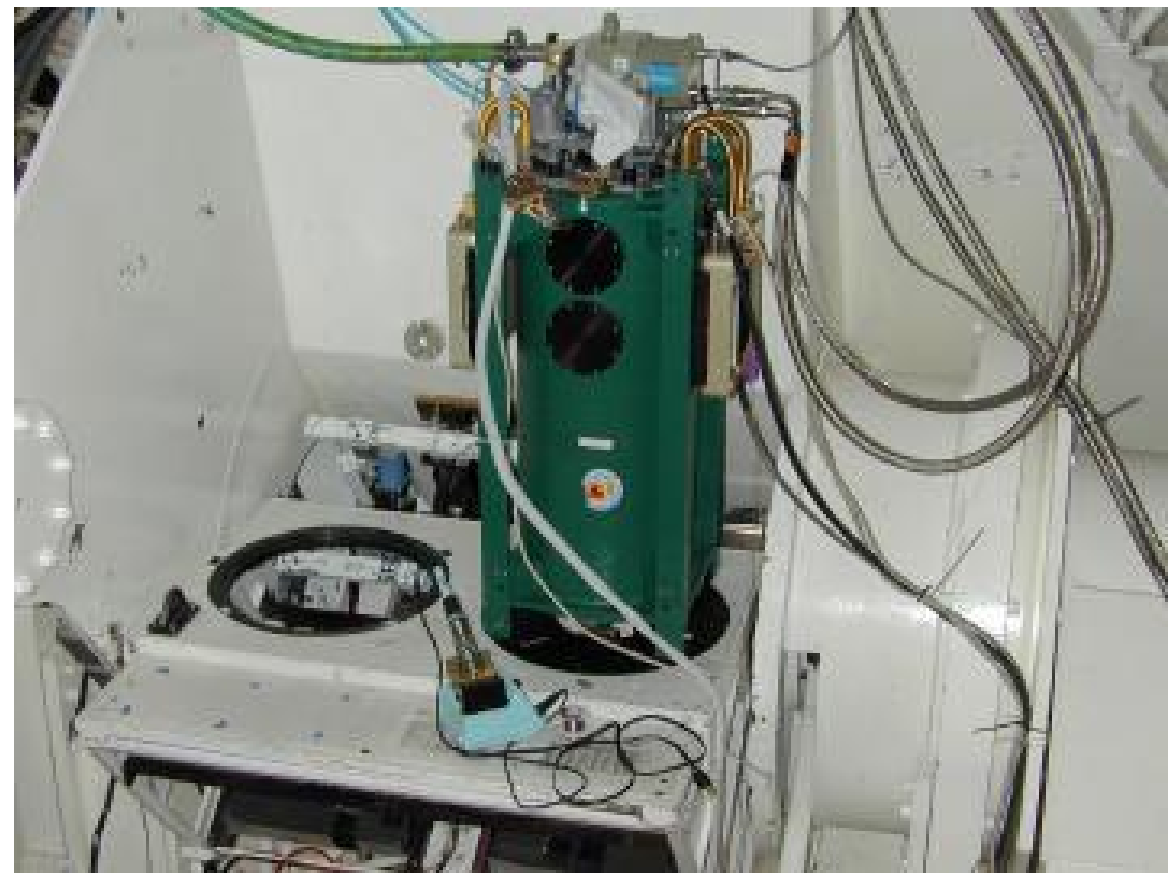

Figure 7. FIBRE in place on the Cassegrain relay focus of the Caltech Submillimeter Observatory on Mauna Kea, Hawai'i.
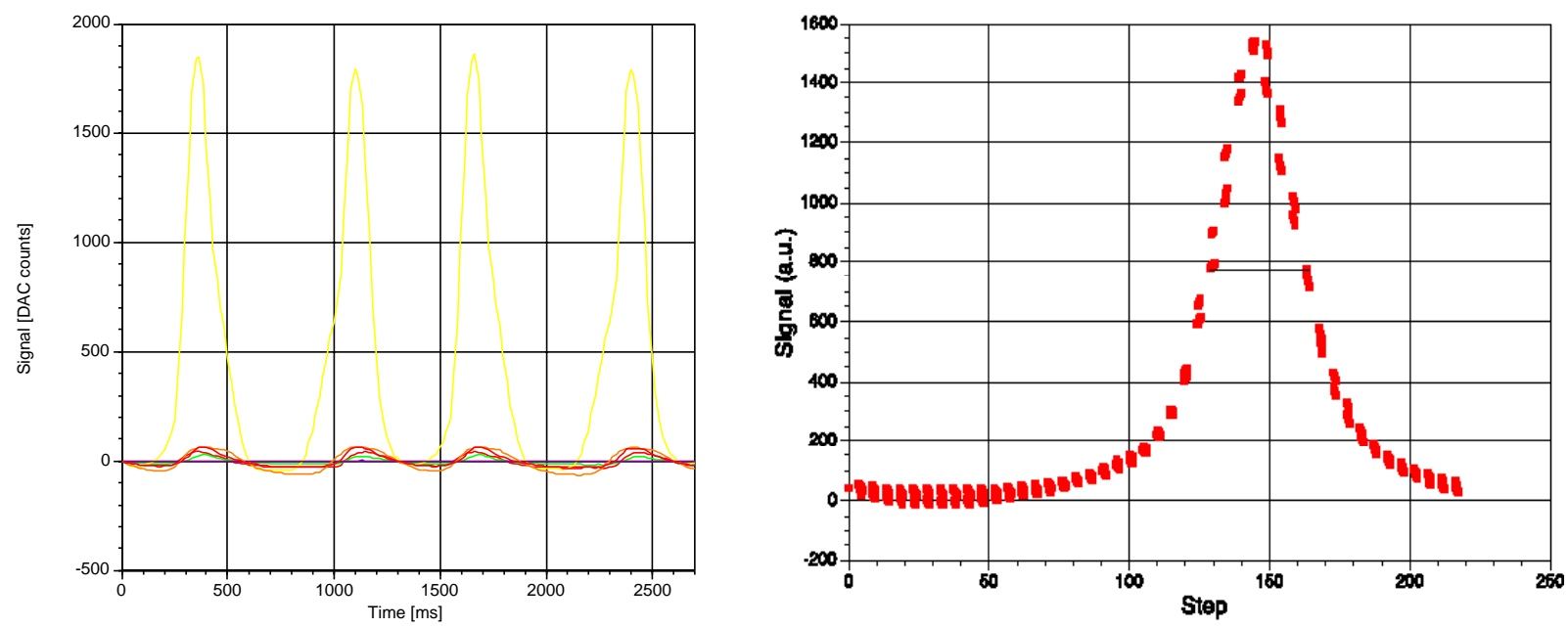

Figure 8. left: The CSO local oscillator seen by several channels while tuning the Fabry-Perot. The spectroscopic performance of FIBRE is demonstrated by the low response of adjacent channels. right: Velocity calibration of a single channel by means of tuning the LO. The resolution is $250 \mathrm{~km} / \mathrm{s}$, corresponding to a resolving power of 1200 . 

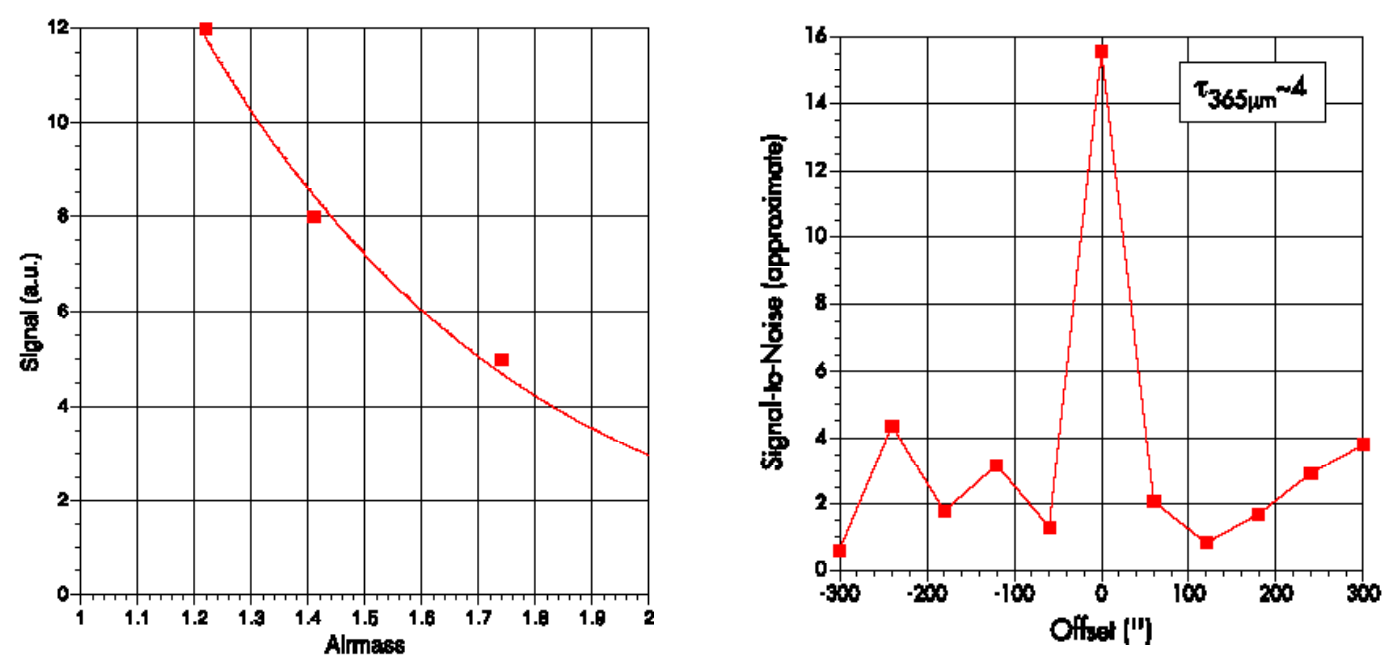

. Figure 9. (left) FIBRE skydip yields $\tau(350 \mu \mathrm{m})=3$.0. (right) FIBRE detects the moon limb at $365 \mu \mathrm{m}$. Each data point corresponds to an on source integration for 3 seconds.

\section{CONCLUSION AND OUTLOOK}

The TES technology and the performance of our time domain SQUID multiplexers has been successfully demonstrated in a sub-mm astronomical observation

bolometers. The performance of FIBRE was close to the predictions. In the meantime we improved the detector design and fabrication [10], the data acquisition system and fixed a problem with the Fabry-Perot motors. With these and other minor design changes we will return to the CSO in July 2004. Weather allowing, we plan to observe [CI] fine structure line emission and rotational CO lines from a number of extragalactic sources. These observations are aimed at gaining new insights into abundances and excitation of the neutral interstellar medium in a variety of galaxy types. Future FIR instruments, such as SPIFI [11], ZEUS [12], the GBT $3 \mathrm{~mm}$ camera [13], and SAFIRE [6], which will fly on the SOFIA airborne observatory, will benefit from our experiences with FIBRE. Ultimately multiplexed ultra-low noise transition edge sensors are a very good candidate to be used in FIR space missions which are aimed to close one of the largest "exploration space gaps" remaining in the field of astronomy.

\section{ACKNOWLEDGEMENTS}

We thank the staff of the Caltech Submillimeter Observatory for their support during observations.

\section{REFERENCES}

1. W.S. Holland, E.I. Robson, W.K.P., Gear, C.R. Cunningham, J.F. Lightfoot, T. Jenness, R.J. Ivison, J.A. Stevens, P.A.R. Ade, M.J. Griffin, W.D. Duncan, J.A. Murphy, D.A. Naylor, "SCUBA: a common-user submillimetre camera operating on theJames Clerk Maxwell Telescope", MNRAS, 303, pp.659-672, 1999

2. D.J. Benford, P. Cox, A. Omont, T.G. Phillips, \& R.G. Mc Mahon, "350 micron dust emission from highredshift objects", ApJL 518 ,pp.65-68, 1999

3. E. Kreysa,.H.P. Gemünd, C.G.T. Haslam, L. Reichertz, E.E. Haller, J.W. Beeman, V. Hansen, A. Sievers, R. Zylka, "Bolometer array development at the Max-Planck Institut für Radioastronomie", SPIE, 3357, pp.319325, 1999

4. K.D. Irwin, “An application of electrothermal feedback for high resolution cryogenic particle detection”, Appl. Phys. Lett., 66, pp.1998-2000, 1995 
5. J.A. Chervenak, K.D. Irwin, E.N. Grossman, J.M. Martinis, C.D. Reintsema, M.E. Huber, "Superconducting multiplexer for arrays of transition edge sensors", Appl. Phys.Letters 74 (26),pp.4043-4045, 1999

6. R.A. Shafer. S.H. Moseley; P.A. Ade,.; D.J. Benford,.; G. Bjoraker, E. Dwek, D.A. Neufeld, F. Pajot, T.G. Phillips, G.J. Stacey, SPIE, 4014, p. 98-108, 2000

7. K.D. Irwin, G.C. Hilton, D.A. Wollman, J.M. Martinis, "Thermal-response time of superconducting transitionedge microcalorimeters", J. Appl. Phys., 83 (8), pp.3978-3985, 1998

8. J.G. Staguhn D.J. Benford, J.A. Chervenak, S.H. Moseley, C.A. Allen, R. Stevenson, W.-T. Hsieh, these proceedings

9. J.G. Staguhn, C.A. Allen, D.J. Benford, J.A. Chervenak, M.M. Freund, S.A. Khan, A.S. Kutyrev, S.H. Moseley, R.A. Shafer, S. Deiker, E.N.Grossman, G.C. Hilton, K.D. Irwin, J.M. Martinis, S.W. Nam, D.A. Rudman, D.A. Wollman.“TES Detector Noise Limited Readout Using SQUID Multiplexers”, 2002, AIP Conf. Proc., 605, pp.321-324

10. C.A. Allen, D.J. Benford, J.A. Chervenak, W.-T. Hsieh, R.A. McClanahan, T.M. Miller, R. Mitchell, S.H. Moseley, J.G. Staguhn, T.R. Stevenson, Nuclear Instruments and Methods in Physics Research, Section A (NIMPA), 520, in press.

11. M.R. Swain, C.M. Bradford, G.J. Stacey, A.D. Bolatto, J.M. Jackson, M.L. Savage, J.A. Davidson, 1998, SPIE, .3354, 480

12. T. Nikola, S. Hailey-Dunsheath, G.J. Stacey, D.J. Benford, S.H. Moseley, J.G. Staguhn, .2003, SPIE 4855, 88

13. D.J. Benford, M.J. Devlin, S.R. Dicker, K.D. Irwin, P.R. Jewell, J. Klein, B.S. Mason, S.H. Moseley, R.D. Norrod, M.P. Supanich, 2004, Nuclear Instruments and Methods in Physics Research, Section A (NIMPA), 520,387 